\title{
CIENCIA\&SALUD
}

\section{Impacto del sedentarismo en la salud mental.}

\section{Impact of sedentarism on mental health.}

Sophia Delgado Villalobos ${ }^{1}$ Cheng Huang Qiu² Leticia González Cordero ${ }^{3}$ Mauricio Castro Sibaja ${ }^{4}$

1,2,3 y 4 Estudiante de Medicina de la Universidad de Ciencias Médicas. Trabajador Independiente, San José Costa Rica.

\section{RESUMEN}

El estilo de vida de las últimas décadas ha generado un incremento en actitudes sedentarias y, junto con el descenso de la actividad física, ha propiciado un efecto negativo en la salud mental. La vida sedentaria aumenta el riesgo de padecer diferentes tipos de trastornos mentales, debido al cual, puede generar alteraciones del sueño, además de un bajo nivel de felicidad. Los padecimientos más frecuentes asociados al sedentarismo son la depresión, ansiedad, baja autoestima, ideación suicida, soledad, estrés y distrés psicológico. El ejercicio físico ha sido demostrado como uno de los tratamientos más importantes para el mejoramiento y manejo de la salud mental en deterioro, ya que tiene beneficios a nivel físico, psicológico y social. Se describen varios de los beneficios que aporta el reducir la ociosidad de las personas, entre los más llamativos están la mejoría en el estado de ánimo, patrón de sueño, función cognitiva, autoestima, calidad de vida, manejo del estrés y reducción del riesgo cardiovascular.

Palabras Clave: sedentarismo, salud mental, adulto joven, bienestar mental, ejercicio.

\section{Cómo citar:}

Delgado Villalobos, S., Huang Qiu, C., González Cordero, L., \& Castro Sibaja, M. Impacto del sedentarismo en la salud mental. Revista Ciencia Y Salud, 6(1), Pág. 81-86. https:// doi.org/10.34192/ cienciaysalud.v6i1.404

Recibido: 30/nov/2021

Aceptado: 10/feb/2021

Publicado: 14/Feb/2022

\section{ABSTRACT}

The lifestyle of the last decades has generated an increase in sedentary behaviours, and paired with the decrease in physical activity, has led to a negative effect on mental health. A sedentary lifestyle is known to increase the risk of suffering from different types of mental disorders, and as a result, it can generate sleep disorders, in addition to a low level of happiness. Among the most frequent conditions that are associated with a sedentary lifestyle are depression, anxiety, low self-esteem, suicidal ideation, loneliness, stress and psychological distress. Practicing physical activity has been shown to be one of the most important treatments for improving and managing mental health deterioration, as it has benefits on a physical, psychological and social level. Several of the benefits attributed to reducing sedentary activities are improvements in daily mood, sleep pattern, cognitive function, self-esteem, quality of life, stress management, as well as significant cardiovascular risk reduction.

Keywords: sedentarism, mental health, young adult, mental wellbeing, exercise. 


\section{Conflicto de Intereses:}

Los autores declaran no tener ningún conflicto de interés.

\section{Financiamiento:}

Los autores declaran no haber recibido ningún financiamiento para la realización de este artículo.

\section{INTRODUCCIÓN}

En las últimas décadas se ha evidenciado que los adultos jóvenes son quienes han tenido un incremento en actividades como el tiempo de vigilia, permanecer sentado o acostado sin realizar ningún tipo de molestia o agotamiento, así como un descenso en la esfuerzo físico en comparación a otros grupos etarios, aumentando su riesgo para desarrollar enfermedades crónicas. El estilo de vida inactivo tiene un efecto negativo sobre el estado mental, puesto que aumenta el riesgo de padecer ansiedad y depresión, disminuyendo los niveles de bienestar emocional. La acumulación de tiempo sedentario también se ha visto vinculado con desórdenes del sueño, los cuales frecuentemente son comorbilidades para padecer de problemas relacionados a la salud mental.[1]

Actualmente se ha encontrado evidencia considerable de la efectividad del estar en constante movimiento como monoterapia en el manejo de la depresión de leve a moderado, ansiedad, alteraciones de la imagen corporal; de la misma manera, funciona como adyuvante en la esquizofrenia, trastornos conversivos y dependencia alcohólica.[2] Por medio de esta revisión bibliográfica se pretende demostrar con datos científicos la importancia de reducir actividades sedentarias en el ámbito de la estabilidad emocional, al igual que definir objetivamente lo que se considera sobre salud mental, así como recalcar la importancia de fomentar hábitos saludables y la importancia de la actividad física para reducir el impacto negativo sobre el bienestar mental.

\section{MATERIALES Y MÉTODOS}

Se realizó una revisión sistemática en donde se tomaron artículos científicos de las siguientes bases de datos: SciELO, Elsevier, PubMed, Dynamed y EBSCOhost. Las palabras claves utilizadas para esta revisión fueron: sedentarismo, salud mental, adulto jóven, bienestar mental y actividad física, y se utilizó el operador booleano "AND" con los términos sedentarismo, salud mental y adulto jóven. Se incluyeron artículos que estuvieran en español o en inglés, además de publicaciones que tuvieran una antigüedad de no más de 5 años. Se excluyeron artículos que fueran publicados por un solo autor, que traten exclusivamente sobre la población adulta mayor y/o pacientes en edad pediátrica. Se obtuvieron 4320 artículos inicialmente y posterior a los criterios de inclusión y exclusión se tomaron en cuenta 141 artículos.

\section{DISCUSIÓN}

\section{Actividades sedentarias}

El sedentarismo o actitudes sedentarias según la Revista Internacional de Nutrición y Actividad física, se definen como actividades que requieren poco gasto de energía, incluyendo el sentarse o acostarse mientras se ve la televisión, jugar con videojuegos, leer, estudiar, escribir o trabajar en la computadora.[1] Se puede relacionar el ejercicio con la cantidad de energía que se consume, por lo que se recomienda cuantificar el esfuerzo en unidades o equivalentes metabólicos (MET) que significa que por cada unidad de oxígeno que se consume por kilogramo de peso corporal en un tiempo específico.[2] Por ejemplo, estar la mayor parte del tiempo sentado o reclinado genera un gasto energético mínimo que corresponde a 1.5 METs, comparado con 6 o 7 METs, los cuales son consumidos en actividad aeróbica y de alto impacto.[2]

A nivel bioquímico, fisiológico y patológico, hay un empeoramiento de la salud cardiometabólica, lo que aumenta la inflamación (Proteína C reactiva e IL-6) y se produce un decaimiento en el estado de ánimo, 


\section{CIENCIA\&SALUD}

incrementando la posibilidad de padecer cualquier enfermedad mental. Otro proceso por el cual se puede explicar el incremento de estas patologías, son las limitaciones en cuanto al movimiento que se ven directamente relacionadas con problemas del sueño propiciando el deterioro de la salud mental. Estos datos contribuyen un excelente aporte para poder ayudar a las personas, tomando en cuenta que pueden ser parte de las estrategias que estimule la reducción del riesgo de padecer estas patologías, evitar que empeoren o bien, incentivar una recuperación más pronta.[3]

Un estudio realizado en Chile, ha relacionado que realizar actividades sedentarias durante el trabajo acorta el tiempo para realizar algún tipo de entrenamiento o deporte, debido a que la mayoría de los trabajos requieren pasar la mayor parte del tiempo sentado, siendo altamente pasivo, lo que genera que el tiempo libre que se puede utilizar para realizar cualquier ejercicio físico, sea utilizado para descansar, de manera que se contribuye al sedentarismo.[4] Otros aspectos que hay que contemplar son la inexistencia de parques, aceras e instalaciones deportivas y recreativas que imparten de manera significativa.[3]

\section{Salud mental}

Desde la perspectiva de la psiquiatría, la cual nos indica que para tener salud mental, no solo abarca la ausencia de alguna enfermedad, sino que también se considera el estado de equilibrio y adaptación ante cualquier situación proporcionando un medio de crecimiento y bienestar individual.[5] De acuerdo con la Organización Mundial de la Salud, la salud mental se define como el estado de bienestar en el cual un individuo es consciente de sus habilidades, es capaz de lidiar con el estrés de la vida diaria, puede trabajar productivamente y es capaz de contribuir a su comunidad.[6]

Los síntomas o trastornos asociados a una salud mental deficiente puede tener una clínica muy variable, entre los más frecuentes son la depresión, ansiedad, baja autoestima, ideación suicida, soledad, estrés y distrés psicológico.[1] Además, se puede observar la aparición de enfermedades, tales como obesidad, hipertensión, diabetes mellitus tipo 2, síndrome metabólico, enfermedades cardiovasculares, cáncer y el incremento en la mortalidad.[5]

\section{Relación del sedentarismo y salud mental}

Se evidenció en investigaciones llevadas a cabo en Estados Unidos que demuestran que los niveles de actividad física insuficientes, aproximadamente 11 horas al día de inactividad, se asocian a un riesgo elevado de decaimiento del estado emocional, así como, al estar esta enfermedad diagnosticada e iniciar con un plan de entrenamiento va a tener un impacto positivo sobre este. Además, se puede observar que la mayoría de las personas con depresión y ansiedad, padecen de alguna otra patología concomitante produciendo mayor sintomatología, una disminución al apego al tratamiento y una muy lenta recuperación. Se demostró que tanto para sedentarismo como para depresión comprenden los mismos factores de riesgo, entre ellos las limitaciones de movimiento, dolor, incomodidad, problemas cognitivos, sueño, baja energía, ansiedad y discapacidad.[7,8]

En cuanto a diversas revisiones sistemáticas realizadas por el International Journal of Behavioral nutrition and Physical Activity, se han encontrado una relación directa entre sintomatología depresiva y sedentarismo relacionado con el uso ocioso de dispositivos electrónicos y adicionalmente se ha sugerido que bajos niveles de tiempo frente a las pantallas digitales se asociaron a un menor nivel de estado depresivo. Según dichos estudios, los síntomas adversos aparecen de dos a tres horas o más de uso diario de pantallas por lo cual establece un punto de corte entre las horas adecuadas frente a dispositivos digitales para reducir los efectos adversos.[1]

Existe evidencia en revisiones de temas realizadas en España que un importante porcentaje de la población sufre en cierto momento de su vida episodios de depresión ya sean moderados o intermedios y en algunos casos graves pueden haber tendencias suicidas; asociado a esto se ha demostrado que un estilo de vida 


\section{CIENCIA\&SALUD}

saludable practicando actividad física está asociado a una reducción sustancial de estos síntomas depresivos. Se ha reflejado que las personas que reducen su ejercicio físico en un determinado periodo de años son más propensos a presentar episodios de desaliento; se ha demostrado que tanto el ejercicio aeróbico como el de fuerza repercuten de manera importante en una reducción de síntomas que limitan una salud mental genuina. Los datos obtenidos indican que realizar actividad física es sinónimo de un aumento en el bienestar subjetivo, traducido en felicidad o placer con la vida, el entorno familiar y el ambiente laboral.[9,10]

Cuadro 1. Beneficios del ejercicio físico en la salud mental y física.[12]

\begin{tabular}{|c|c|}
\hline Estado de ánimo & $\begin{array}{l}\text { Mejora el estado de ánimo al incrementar la circulación } \\
\text { sanguínea cerebral } \\
\text { Ejerce influencia sobre el eje hipotálamo-hipófiso-adrenal } \\
\text { sobre la respuesta fisiológica al estrés } \\
\text { Contrarresta el estado de ánimo negativo, al mejorar la } \\
\text { autoestima y la función cognitiva }\end{array}$ \\
\hline Patrón de sueño & $\begin{array}{c}\text { Alternativa no farmacológica como tratamiento en } \\
\text { trastornos del sueño }\end{array}$ \\
\hline Función cognitiva & $\begin{array}{l}\text { Mejora la cognición en adultos mayores con riesgo de } \\
\text { demencia es una regulación de la función vascular cerebra } \\
\text { y la perfusión cerebral. } \\
\text { Adultos mayores genera una mejoría tanto en la función } \\
\text { cognitiva como en el estado de ánimo } \\
\text { Aumento del flujo sanguíneo a áreas críticas del sistema } \\
\text { nervioso central relacionadas con el aprendizaje y otras } \\
\text { funciones mentales superiores }\end{array}$ \\
\hline Autoestima & $\begin{array}{l}\text { Alivio de síntomas de baja autoestima y aislamiento social } \\
\text { Mejora la autoestima física y la percepción de la condición } \\
\text { física }\end{array}$ \\
\hline Calidad de vida & $\begin{array}{l}\text { Efecto positivo sobre la calidad de vida } \\
\text { Mejora la calidad de vida en relación a la salud }\end{array}$ \\
\hline Afrontamiento del estrés & Afrontamiento efectivo y positivo \\
\hline Aptitud cardiovascular & $\begin{array}{c}\text { Mejora la salud cardiovascular, importante en pacientes } \\
\text { en tratamiento farmacológico }\end{array}$ \\
\hline Reducción del estrés & $\begin{array}{l}\text { Reduce el estrés } \\
\text { Aumenta la energía y la alerta mental } \\
\text { Aumenta la resistencia corporal general }\end{array}$ \\
\hline
\end{tabular}

Asimismo, el ejercicio físico presenta una eficacia significativa en el tratamiento de episodios de ansiedad, depresión y el mejoramiento de la autoestima, y en periodos tan cortos como diez días ya se pueden observar mejoras; no obstante, para que los efectos que se producen sean a largo plazo se debe de tener un estilo de vida activo y no necesariamente una intervención directa. Aunado a esto de un estudio retrospectivo que se llevó a cabo de Gran Bretaña, entre otras conclusiones se determinó que los adultos que realizaban deporte periódicamente, fueron niños más activos, más extrovertidos y llevaron una vida más saludable en su adolescencia. También de un estudio prospectivo con 30.000 sujetos con una extensión de 14 años se logró determinar un considerable beneficio al realizar actividad física en el tiempo libre así como una 


\section{CIENCIA\&SALUD}

disminución importante en la mortalidad, por lo que se determinó una relación inversa entre la actividad física que realizaban los sujetos frente a la mortalidad de estos sujetos.[9,11]

\section{Hábitos de mejora}

Los beneficios que trae consigo reducir el sedentarismo en la población joven son el mantenimiento de un peso saludable, mejora en la forma física, en el aprendizaje, la atención, el comportamiento y el rendimiento escolar junto a estos facilita el desarrollo de habilidades tanto sociales como del lenguaje. Así mismo la OMS menciona que los adultos que realizan la cantidad de actividad física recomendada de 150 minutos de ejercicio moderado a la semana o 75 minutos de actividad física aeróbica vigorosa corren un menor riesgo de padecer depresión y otras patologías crónicas. [3,13]

Actualmente el sedentarismo sigue siendo uno de los más grandes problemas en países del primer mundo y especialmente en aquellos en vías de desarrollo puesto que esto constituye uno de los principales factores de riesgo para padecer enfermedades que mayor mortalidad presenta a nivel global. [14]

Estudios han reportado una relación a la inversa entre el tiempo realizando actividad aeróbica y depresión; en el cual cabe resaltar que aquellas personas que reportaron un nivel mayor de movilidad también reportaron el nivel más alto de bienestar general. La actividad física es una herramienta importante tanto para el tratamiento como la prevención de enfermedades mentales, y si bien no es superior a los otros distintos tipos de tratamientos, es un excelente complemento para estos.[7,15]

\section{CONCLUSIÓN}

Al realizarse la presente revisión bibliográfica se evidencia que la salud mental es parte esencial de la integridad de los seres humanos en cuanto a materia de salud se refiere, por ello al ver el evidente incremento de las actividades sedentarias en los últimos años, representa una llamada de atención, pues esto ha repercutido sobre las diferentes poblaciones que componen la sociedad actual, en particular la población de adultos jóvenes.

De la mano con el aumento del sedentarismo se ha observado un aumento en la población de adultos jóvenes que ven afectada su salud mental al desarrollar padecimientos psiquiátricos como trastornos de ansiedad y depresión, así como su capacidad para sobrellevar los mismos. Es aquí donde recae la importancia del fomento de la actividad física como un complemento del manejo de estas patologías y método preventivos de la aparición de las mismas, argumento que se observó a lo largo de la revisión.

Es destacable hacer un énfasis según lo plasmado en esta revisión, que la actividad física es una excelente herramienta en el afrontamiento de situaciones de estrés que puedan presentarse como desencadenante en el desarrollo de trastornos psiquiátricos que afecten el desenvolvimiento social de los pacientes y su integridad personal.

\section{REFERENCIAS BIBLIOGRÁFICAS}

1. Hoare E, Milton K, Foster C. The associations between sedentary behaviour and mental health among adolescents: a systematic review. Int J Behav Nutr Phys. 2016;13(1):1-22. DOI: 10.1186/s12966016-0432-4

2. Romero T. Hacia una definición de Sedentarismo. Rev Chil Cardiol. 2009 Dic ; 28( 4): 409-413. DOI: $10.4067 /$ S0718-85602009000300014

3. Ambroa de Frutos G. Impacto del sedentarismo sobre la práctica de actividad física y la salud. REEFD. DOI: 2016;(412):33-44. 


\section{CIENCIA\&SALUD}

4. Carrazana V. El concepto de salud mental en psicología humanista-existensial. UCBSP. 2003; (1):1-19.

5. Salas C, Cristi-Montero C, Fan Y, Durán E, Labraña A, Martínez M et al . Ser físicamente activo modifica los efectos nocivos del sedentarismo sobre marcadores de obesidad y cardiometabólicos en adultos. Rev. méd. Chile. 2016 Nov; 144 (11): 1400-1409. DOI: 10.4067/S0034-98872016001100005.

6. World Health Organization. 2004. Promoting Mental Health. https://www.who.int/mental_health/ evidence/en/promoting_mhh.pdf

7. Ellingson L, Meyer J, Shook R, Dixon P, Hand G, Wirth M, Paluch A, Burgess S, Hebert J, Blair S. Changes in sedentary time are associated with changes in mental wellbeing over 1 year in young adults. Prev. Med. Rep. 2018;(11):274-281.DOI: 10.1016/j.pmedr.2018.07.013

8. Del Valle Soto M, Prieto Saborit J.A, Nistal Hernandez P, Martinez Suarez P.C, Ruiz Fernandez L. Impacto de las estrategias de ejercicio físico en la CVRS de adultos sedentarios. (2016). RIMCAFD. 16 (64) , pp. 739-756. DOI: 10.15366/rimcafd2016.64.008

9. Márquez S, Rodriguez J, De Abajo S. Sedentarismo y salud: efectos beneficiosos de la actividad física. Apunts. Educ. Fís. Deporte. 2006; 12-24.

10. De la Cruz E, Moreno M, Pino J, Martinez R. Actividad física durante el tiempo libre y su relación con algunos indicadores de salud mental en España. Scielo.org.mx. Salud Ment. vol.34 no.1 México ene./feb. 2011

11. Caracuel J, Arbinaga F. Repercusión del ejercicio físico sobre la salud. (2012). Repercusión del ejercicio físico sobre la salud. Apuntes de Psicología, 30 (1-3), 547-554. Idus.us.es.

12. Torales J, Almiron M, Gonzalez I, Gomez N, Ortiz R, Ibarra V. Ejercicio físico como tratamiento adyuvante de los trastornos mentales. Una revisión narrativa. FCM-UNA. 2018, (51) 27-32. DOI: 2018.051(03)27-032

13. Cantón E. DEPORTE, SALUD, BIENESTAR Y CALIDAD DE VIDA. Cuad. psicol. deporte. 2001

14. Stanton R, Happell B, Reaburn P. The mental health benefits of regular physical activity, and its role in preventing future depressive illness. Nursing: Research and Reviews. 2014;4:45-53 DOI: 10.2147/ NRR.S41956

15. Stubbs B, Vancampfort D, Firth J, Schuch F, Hallgren M, Smith L, Gardner B, Kahl K, Veronese N, Solmi M, Carvalho A, Koyanagi A. Relationship between sedentary behavior and depression: A mediation analysis of influential factors across the lifespan among 42,469 people in low- and middleincome countries. J. Affect. Disord. 2018; 231-238 DOI: 10.1016/j.jad.2017.12.104. 